\title{
Progressive Diffuse Pulmonary Interstitial Opacities due to Complications of Pulmonary Tumor Emboli: An Autopsy Case Report
}

\author{
Yutaka Yoshii $^{1,2}$, Yoshinori Kawabata ${ }^{3}$, Noboru Takayanagi ${ }^{1}$, \\ Jun Araya $^{2}$, Kazuyoshi Kuwano ${ }^{2}$ and Yutaka Sugita ${ }^{1}$
}

\begin{abstract}
A 76-year-old man complaining of exertional dyspnea was admitted to our hospital. Chest computed tomography revealed bilateral diffuse ground-glass opacities and small nodules. A transbronchial lung biopsy revealed tumor cell emboli in the pulmonary arteries. The patient was diagnosed with gastric adenocarcinoma using an endoscopic stomach biopsy; however, the interstitial opacities progressively worsened and he died of acute respiratory failure. An autopsy revealed extensive pulmonary tumor embolisms (PTE) with associated ischemic damages, e.g., infarctions, alveolar wall thickening with cuboidal metaplasia, hemorrhage, and diffuse alveolar damage. The ground-glass opacities in the chest computed tomography findings appear to correlate with the pathological ischemic changes associated with PTE.
\end{abstract}

Key words: diffuse interstitial opacities, pulmonary tumor embolism, pulmonary infarction

(Intern Med 54: 955-960, 2015)

(DOI: 10.2169/internalmedicine.54.3476)

\section{Introduction}

Pulmonary tumor embolism (PTE) is a classic manifestation of cancer metastasis to the lung. The occlusion of pulmonary microvasculature by tumor cells accompanied by thrombi typically produces a subacute and progressive clinical presentation resembling thromboembolic disease (1). Although pulmonary infarction and hemorrhaging are typical pathological findings associated with PTE (2), a correlation between the pathological findings and the radiological findings remains to be determined.

We herein report the case of a 76-year-old man presenting with bilateral ground glass opacities (GGO) resembling interstitial pneumonia on chest computed tomography (CT). On the autopsy, GGO appeared to be related to hemorrhaging, interstitial pneumonia-like lesions, and diffuse alveolar damage (DAD). Thus, based on the autopsy, we posthumously diagnosed the patient with PTE.

\section{Case Report}

A 76-year-old man presented to a local clinic with a dry cough and exertional dyspnea. Because his symptoms were refractory and progressively worsened over the course of a month, he was admitted to our hospital for further examination. His body weight had decreased by $10 \mathrm{~kg}$ over the previous 3 months. The physical examination on admission revealed no significant abnormalities, and the superficial lymph nodes were not palpable. Laboratory tests revealed anemia and coagulation abnormalities (hemoglobin concentration, $12.2 \mathrm{~g} / \mathrm{dL}$; prothrombin time-international normalized ratio, 1.36; activated partial thromboplastin time, 35.9 $\mathrm{s}$; and fibrinogen level, $697 \mathrm{mg} / \mathrm{dL}$ ). The level of surfactant protein-D (SP-D) was elevated $(151.1 \mathrm{ng} / \mathrm{mL})$, whereas the level of Krebs von den Lungen-6 (KL-6) was within the normal range. The laboratory tests also indicted elevated levels of C-reactive protein $(4.7 \mathrm{mg} / \mathrm{dL})$ and carcinoembry-

\footnotetext{
${ }^{1}$ Department of Respiratory Medicine, Saitama Cardiovascular and Respiratory Center, Japan, ${ }^{2}$ Division of Respiratory Diseases, Department of Internal Medicine, The Jikei University School of Medicine, Japan and ${ }^{3}$ Division of Diagnostic Pathology, Saitama Cardiovascular and Respiratory Center, Japan

Received for publication June 16, 2014; Accepted for publication September 28, 2014

Correspondence to Dr. Yutaka Yoshii, y.yoshii@jikei.ac.jp
} 


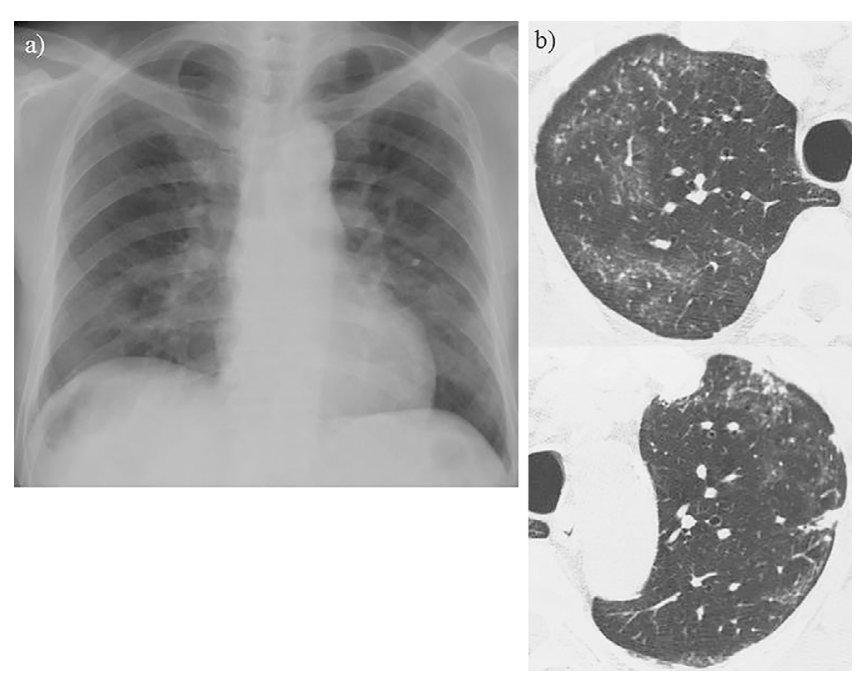

Figure 1. Radiological findings on admission. (a) A chest Xray showed bilateral ground-glass opacities. (b) Chest computed tomography showed small nodules and linear opacities around and inside the areas of ground-glass opacity which were predominantly located in the peripheral lung field and spared the subpleural space.

onic antigen $(6.8 \mathrm{ng} / \mathrm{mL})$. Moreover, we did not observe any immunological abnormalities, such as aberrant leukocyte counts, unusual immunoglobulin or complement levels, or autoantibodies. A blood gas analysis in the atmosphere of room air revealed hypoxia $\left(\mathrm{pH}, 7.44 ; \mathrm{PaO}_{2}, 66.2\right.$ Torr; $\mathrm{PaCO}_{2}, 36.7$ Torr).

A chest X-ray showed bilateral GGO, and a chest CT demonstrated small nodules and linear opacities around and inside GGO which were predominantly located in the peripheral lung field and spared the subpleural space (Fig. 1). A sputum culture was negative for bacteria, and no abnormal findings were detected on the sputum cytology. Based on the radiological findings, we initially considered diffuse alveolar hemorrhage or eosinophilic pneumonia as the provisional diagnosis; however, these were excluded due to the absence of hemosiderin-laden macrophages and eosinophils in the bronchoalveolar lavage fluid. A transbronchial lung biopsy (TBLB) specimen from right $\mathrm{B}^{1}$ demonstrated marked alveolar wall thickening with cuboidal metaplasia (Fig. 2a, b). Accordingly, we tentatively diagnosed the patient with interstitial pneumonia of unknown cause. We started the patient on oral prednisolone $(30 \mathrm{mg} / \mathrm{day})$ for the hypoxia symptoms. However, the second CT examination on day 26 of admission showed deterioration of the abnormal shadows (Fig. 3). GGO and linear opacities had extended, and new cavitary nodular lesions were seen. Thus, we suspected a secondary fungal infection and discontinued prednisolone therapy.

BFS was repeated to obtain sufficient material to confirm the pathological diagnosis. The histopathological examination of the specimens from the second TBLB from right $\mathrm{B}^{5}$ and $\mathrm{B}^{8}$ demonstrated microemboli composed of cancer cells inside the pulmonary artery branches; this problem was also accompanied by mild alveolar wall thickening in the surrounding lung (Fig. 2c, d). During the histopathological examination, no obvious intraluminal hemorrhaging was detected in either the first or second TBLB specimens. Gastrointestinal endoscopy was performed to elucidate the primary adenocarcinoma site. Converging folds were detected endoscopically, and a stomach biopsy confirmed gastric adenocarcinoma (Fig. 4a). Thus, we diagnosed the patient with PTE originating from gastric adenocarcinoma. A right heart catheterization excluded pulmonary hypertension (mean pulmonary arterial pressure was $21 \mathrm{mmHg}$ ).

Shortly after the diagnosis of PTE, the patient suffered from acute onset respiratory failure and his symptoms rapidly deteriorated. A third CT examination on day 39 of admission showed an extension of non-segmental GGO accompanied by multiple cavitary lesions (Fig. 3). The patient died on day 40 of admission due to a worsening of his respiratory failure.

An autopsy examination identified the primary diagnosis as advanced gastric adenocarcinoma located on the lesser curvature of the upper body (Fig. 4b). Concomitant multiple metastases were present in the liver, lungs, and lymph nodes. We also performed a detailed evaluation of the pulmonary lesions associated with PTE. Three different types of pulmonary metastasis were detected, including massive tumor emboli with organized thrombi obstructing muscular pulmonary arteries and arterioles, tiny hematogenous metastatic nodules, and lymphogenous spread.

Gross and microscopic changes associated with massive tumor emboli were present as various stages of whitish anemic infarctions and small yellowish infarctions (Fig. 5a, b). Several interstitial pneumonia-like lesions of up to one lobule in size were histologically observed. In general, these lesions were adjacent to the infarction sites and were composed of edematous/myxomatous or fibrous thickening of the alveolar wall (Fig. 6a, b), slight infiltration by inflammatory cells, cuboidal metaplasia (Fig. 6c), and centered capillaries (Fig. 6d). In addition to cord-like and nodular fibrosis (Fig. 5a, b), an alveolar wall hemorrhage (Fig. 5d), a focal intraluminal hemorrhage with hemosiderosis, diffuse alveolar damage (DAD; Fig. 4c), and cavitary lesions (Fig. 5c) were noted. Most cavitary walls were composed of infarction accompanied by bacterial and fungal infections; the fungal infection typically involved Aspergillus spp.

Therefore, the autopsy findings appeared to correlate with the CT findings. The bilateral diffuse GGO on admission was assumed to correspond to the interstitial pneumonia-like lesions. The nodular lesions and linear opacities on admission may represent the organized infarction and cord-like fibrosis. Therefore, cavitary lesions may represent the necrosis related to bacterial and fungal infections and the infarctions due to tumor emboli. Furthermore, the terminal extension of the non-segmental GGO appeared to reflect mainly DAD. 


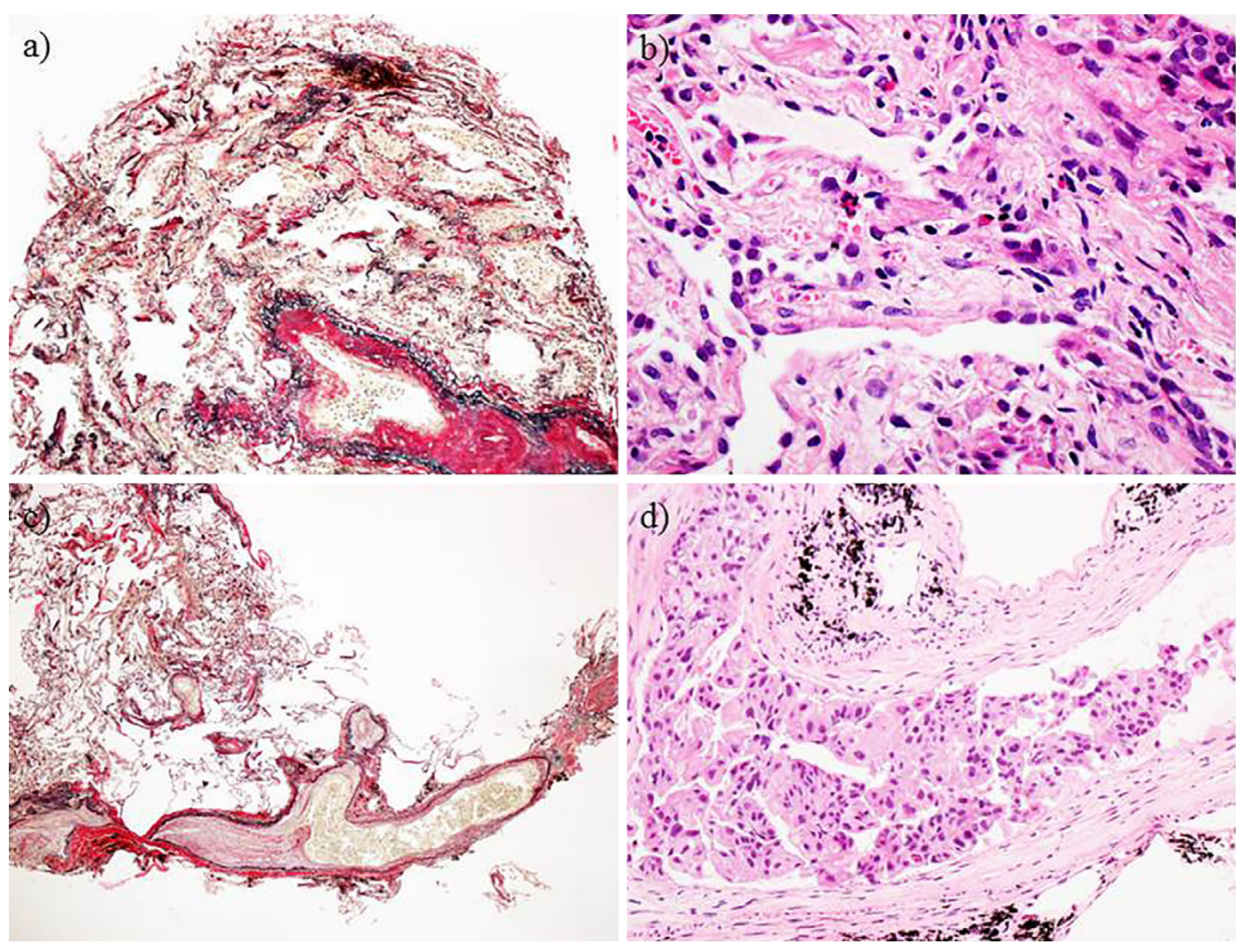

Figure 2. Results of the transbronchial lung biopsy (TBLB). (a) A low magnification view of the alveolar wall thickening [Elastic van Gieson staining (EvG), 4× magnification]. (b) A high magnification view of the alveolar-wall thickening with cuboidal cell metaplasia [Hematoxylin and Eosin (H\&E) staining, 20x magnification]. (c) A low magnification view of tumor emboli and a forming thrombus in the muscular pulmonary artery (EvG, 4× magnification). (d) A high magnification view of microemboli composed of cancer cells in the pulmonary artery (H\&E staining, 20x magnification).

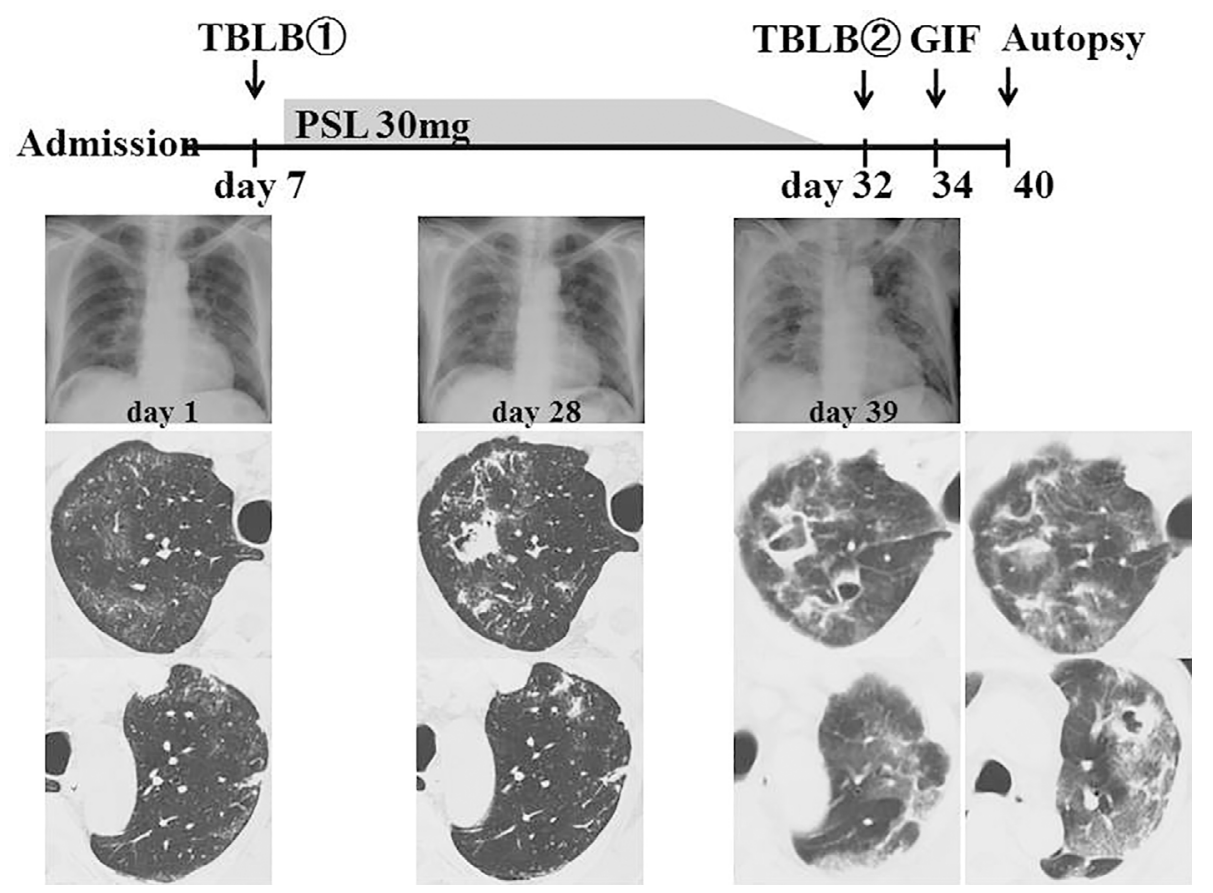

Figure 3. The clinical course. Chest computed tomography showed deterioration of the abnormal shadows. 

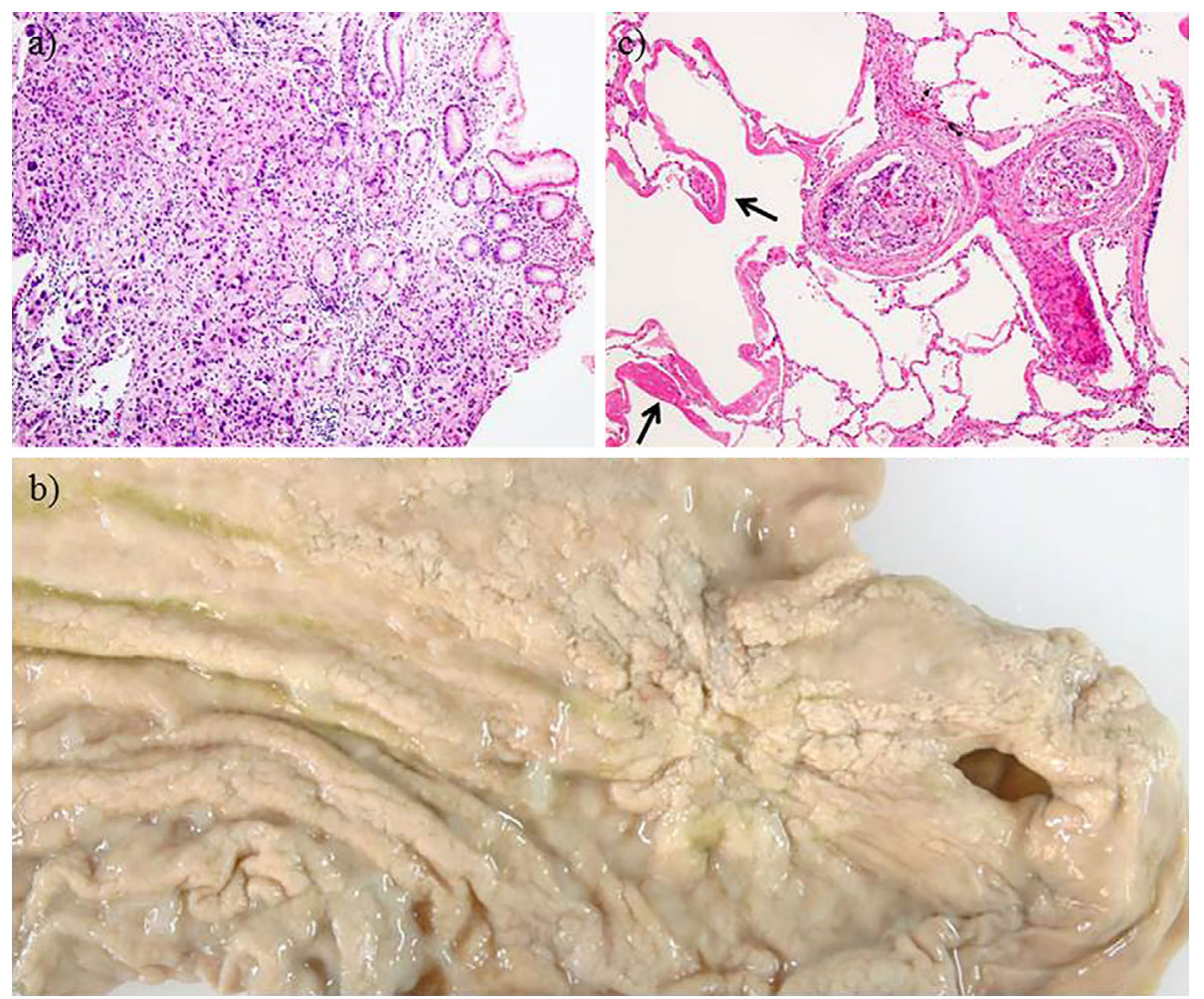

Figure 4. Gross pathological and microscopic findings of the stomach and lung. (a) The stomach biopsy showed adenocarcinoma [Hematoxylin and Eosin (H\&E) staining, 10x magnification]. (b) Gastric cancer was seen in the lesser curvature of the upper body at autopsy. (c) Hyaline membrane (arrows), which is a representative finding in diffuse alveolar damage (DAD), and tumor emboli on the alveolar wall during autopsy is shown (H\&E staining, 10× magnification).
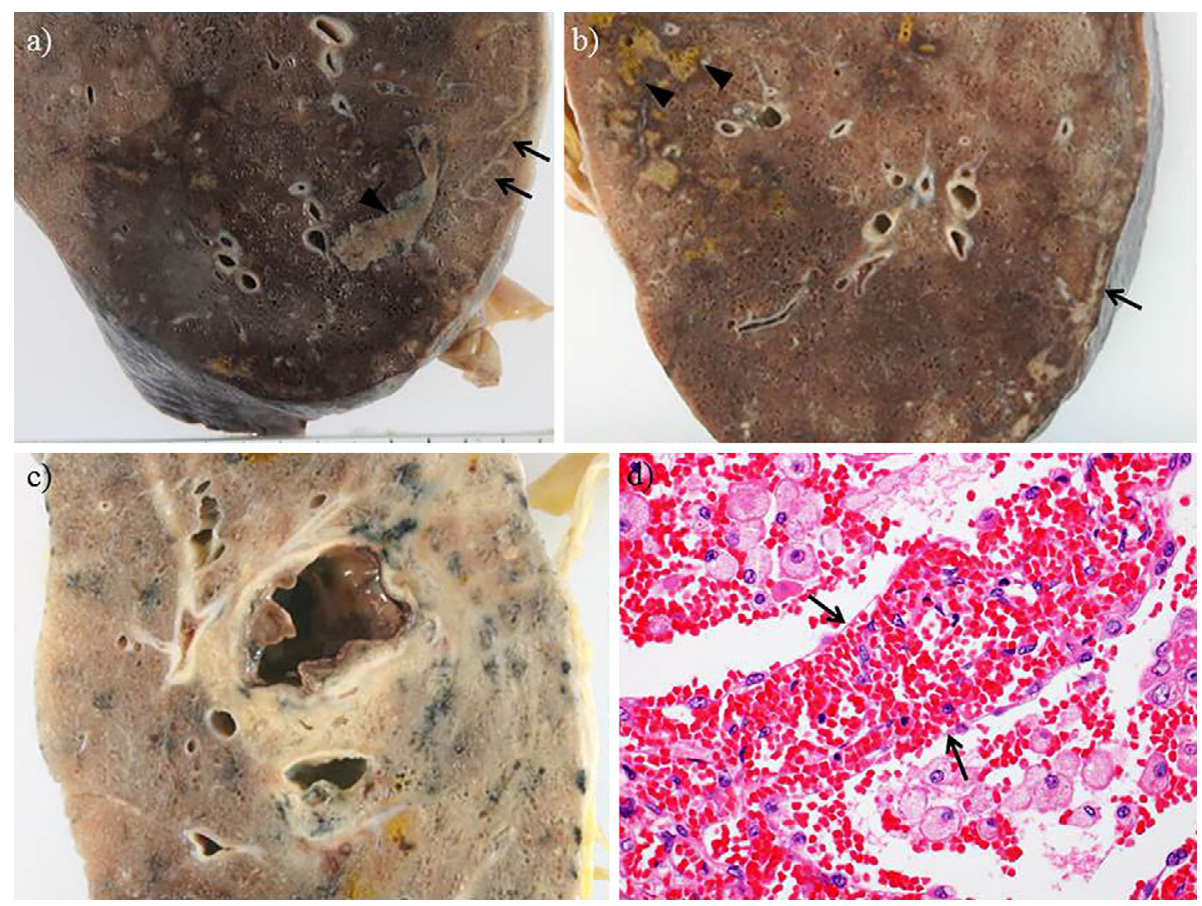

Figure 5. Gross pathological and microscopic findings of the lung at autopsy. The cut surface of the lung showed (a) a pulmonary infarction (arrowhead) and linear fibrosis (arrows) in diffuse consolidation, (b) a yellowish infarction (arrowheads) and linear fibrosis (arrow) in diffuse consolidation, and (c) cavitation. (d) Microscopic findings showed an alveolar wall hemorrhage (between arrows) (H\&E staining, 40× magnification). 
a)
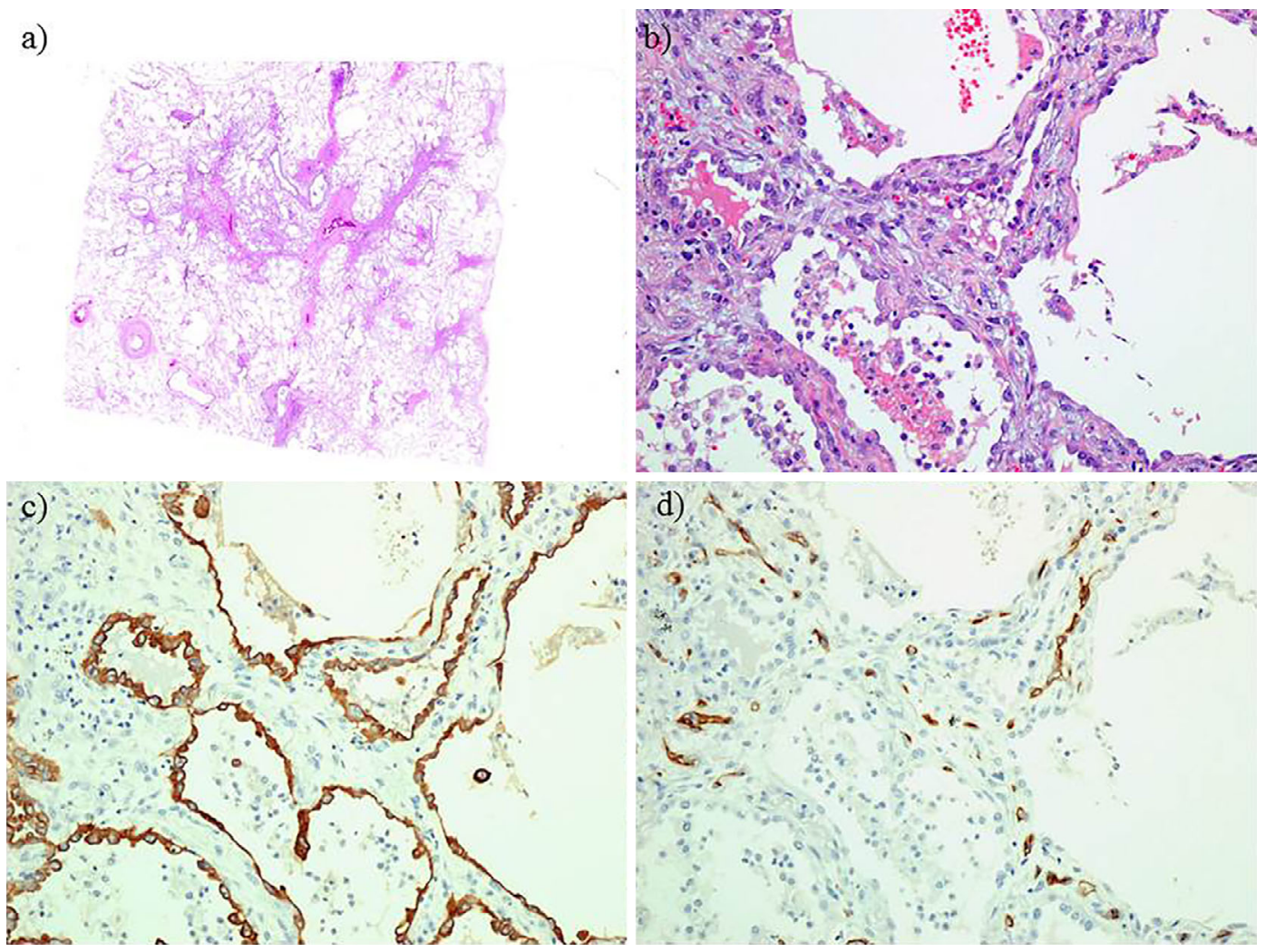

Figure 6. Lung microscopy at autopsy: a lobular interstitial pneumonia-like lesion. (a) A panoramic view of a lobule-sized lesion in the center [Hematoxylin and Eosin (H\&E) staining]. (b) Edematous/ myxomatous thickening of the alveolar wall with cuboidal metaplasia is shown (H\&E staining, 20x magnification). (c) Diffuse cuboidal cell metaplasia by immunostaining using pan-keratin is shown (keratin, 20x magnification). (d) Alveolar capillaries located in the center of the alveolar wall without attachment to the surface of the alveolar wall is shown (immunostaining for CD34, 20x magnification).

\section{Discussion}

In the present case of advanced gastric cancer with PTE, the pathological findings (acute ischemic lung damages due to PTE) correlated with the radiological findings (diffuse interstitial opacities on CT). PTE is associated with a coagulation abnormality related to cancer progression and is generally diagnosed on post-mortem examination $(3,4)$. PTE mainly involves muscular arteries and causes intimal fibrosis $(5,6)$. Pulmonary infarction and hemorrhaging are caused by insufficient pulmonary arterial blood flow resulting from thromboembolism and tumor cell emboli $(3,7,8)$.

In the present case, various pathological findings were observed in addition to infarction and focal hemorrhaging, including alveolar wall hemorrhages, linear/nodular fibrosis, and interstitial pneumonia-like lesions. Moreover, an alveolar wall hemorrhage was seen in association with pulmonary infarction, and cord-like and nodular fibrosis were frequently connected to the infarction walls. These characteristic findings can be attributed to ischemia due to insufficient pulmonary arterial blood flow associated with PTE.

Intriguingly, consistent with the findings of the initial TBLB samples, interstitial pneumonia-like lesions were lobule-sized and showed edematous or fibrous thickening with scanty inflammatory cells and separation between the epithelial and endothelial basement membranes. We have previously reported the association between this type of basement membrane separation in the alveolar wall and pulmonary infarction caused by lung tumors (9). These findings differ from non-specific interstitial pneumonia, which is usually diffuse and accompanied by moderate-to-severe inflammatory cell infiltration of the alveolar wall with luminal organization $(10,11)$. Therefore, the interstitial pneumonialike lobule-sized lesions that were observed in the present case may represent the pathological changes reflecting tissue ischemia, but they are not associated with primary inflammatory reaction (i.e., idiopathic, collagen vascular disease, and drug induced). Furthermore, the ineffectiveness of the steroid treatment may support the notion of noninflammatory nature of interstitial pneumonia-like lesions.

DAD pattern is a known complication of pulmonary infarction (12). A previous report examining 318 autopsied cases from patients who died of various cancers (excluding lung cancer) revealed that four of the 12 PTE patients presented DAD pattern during the lung examination (13). Furthermore, in our study involving patients with DAD pattern associated with subclinical idiopathic pulmonary fibrosis, 
approximately $10 \%$ patients had pulmonary thromboemboli and disseminated intravascular coagulation (14) indicating that widespread PTE is a possible risk factor of DAD in the present case. In addition, we speculate that severe fungal and bacterial infection of cavitary nodular lesions is also involved in the mechanism of DAD development. These infections may therefore be attributed to the patient's weakened immune system due to steroid treatment and/or cancer progression.

In conclusion, we herein reported on the pathologicalradiological correlation between the autopsy findings of acute ischemic lung damage due to PTE and pulmonary interstitial opacities on CT in a case of advanced gastric cancer. Clinicians should consider acute ischemic lung damage as a differential diagnosis of steroid-refractory progressive interstitial opacities, especially in cases with malignant tumors.

The authors state that they have no Conflict of Interest (COI).

\section{Acknowledgement}

We would like to thank Dr. Thomas V. Colby (Mayo Clinic, Arizona) for his valuable help and thoughtful suggestions.

\section{References}

1. Roberts KE, Hamele-Bena D, Saqi A, Stein CA, Cole RP. Pulmonary tumor embolism: a review of the literature. Am J Med 115: 228-232, 2003.

2. Rossi SE, Goodman PC, Franquet T. Nonthrombotic pulmonary emboli. AJR Am J Roentgenol 174: 1499-1501, 2000.

3. Fraser RS, Muller NL, Colman NC, Pare PD. Thrombosis and thromboembolism. In: Fraser and Pare's Diagnosis of Diseases of the Chest. 4th ed. Fraser RS, Muller NL, Colman NC, Pare PD, Eds. Saunders, Philadelphia, 1999: 1773-1843.

4. Webb WR, Muller NL, Naidich DP. Pulmonary hypertension and pulmonary vascular disease. In: High-Resolution CT of the Lung. Webb WR, Muller NL, Naidich DP, Eds. LWW, Philadelphia, 2009: 555-584.
5. Shields DJ, Edwards WD. Pulmonary hypertension attributable to neoplastic emboli: an autopsy study of 20 cases and a review of the literature. Cardiovasc Pathol 1: 279-287, 1992.

6. Ahmed AA, Heller DS. Fatal pulmonary tumor embolism caused by chondroblastic osteosarcoma: report of a case and review of the literature. Arch Pathol Lab Med 123: 437-440, 1999.

7. Corrin B, Nicholson AG. Vascular disease. In: Pathology of the Lungs. 2nd ed. Churchill Livingstone, Philadelphia, 2011: 401458.

8. Groshong SD, Tomashefski JF, Cool CD. Pulmonary vascular disease. In: Dail and Hammar's Pulmonary Pathology, Vo.1. Nonneoploastic Lung Disease. 3rd ed. Tomashefski JF, Cagle PT, Farver CF, Fraire AE, Eds. Springer-Verlag, New York, 2009: 1032-1187.

9. Kawabata Y, Hoshi E, Takayanagi N, Sugita Y. Pathological study of the natural history of pulmonary infarction mainly seen in lung tumors--pulmonary infarction begins with alveolar wall bleeding. Nihon Kokyuki Gakkai Zasshi 47: 851-857, 2009 (in Japanese, Abstract in English).

10. American Thoracic Society, European Respiratory Society. American Thoracic Society/European Respiratory Society International Multidisciplinary Consensus Classification of the Idiopathic Interstitial Pneumonias. This joint statement of the American Thoracic Society (ATS), and the European Respiratory Society (ERS) was adopted by the ATS board of directors, June 2001 and by the ERS Executive Committee, June 2001. Am J Respir Crit Care Med 165: 277-304, 2002.

11. Travis WD, Costabel U, Hansell DM, et al. An official American Thoracic Society/European Respiratory Society statement: Update of the international multidisciplinary classification of the idiopathic interstitial pneumonias. Am J Respir Crit Care Med 188: 733-748, 2013.

12. Parambil JG, Savci CD, Tazelaar HD, et al. Causes and presenting features of pulmonary infarctions in 43 cases identified by surgical lung biopsy. Chest 127: 1178-1183, 2005.

13. Tamura A, Matsubara O. Pulmonary tumor embolism: relationship between clinical manifestations and pathologic findings. Nihon Kyobu Shikkan Gakkai Zasshi (Japanese Journal of Thoracic Diseases) 31: 1269-1278, 1993 (in Japanese, Abstract in English).

14. Kawabata Y, Fukushima K, Uchiyama T, Sugita H, Kimura B. A focal usual interstitial pneumonia lesion: an important risk factor in diffuse alveolar damage--acute exacerbation of a focal usual interstitial pneumonia patient. Nihon Kokyuki Gakkai Zasshi 39: 316-321, 2001 (in Japanese, Abstract in English).

(C) 2015 The Japanese Society of Internal Medicine http://www.naika.or.jp/imonline/index.html 